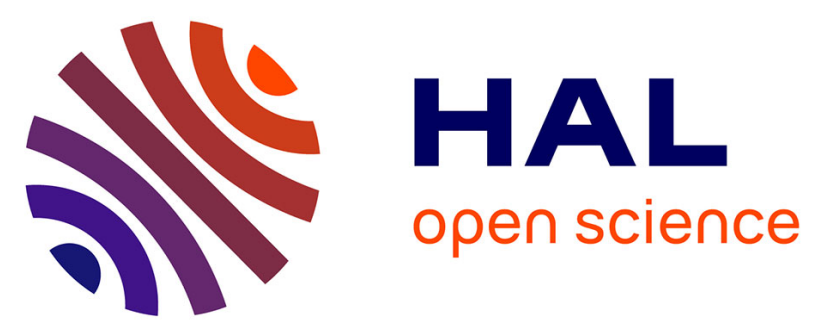

\title{
Asymptotic performance of the Low Rank Adaptive Normalized Matched Filter in a large dimensional regime
}

Alice Combernoux, Frédéric Pascal, Guillaume Ginolhac, Marc Lesturgie

\section{To cite this version:}

Alice Combernoux, Frédéric Pascal, Guillaume Ginolhac, Marc Lesturgie. Asymptotic performance of the Low Rank Adaptive Normalized Matched Filter in a large dimensional regime. 40th IEEE International Conference on Acoustics, Speech and Signal Processing (ICASSP 2015), Apr 2015, Brisbane, Australia. pp.2599 - 2603, 10.1109/ICASSP.2015.7178441 . hal-01226381

HAL Id: hal-01226381

https://hal.univ-grenoble-alpes.fr/hal-01226381

Submitted on 9 Nov 2015

HAL is a multi-disciplinary open access archive for the deposit and dissemination of scientific research documents, whether they are published or not. The documents may come from teaching and research institutions in France or abroad, or from public or private research centers.
L'archive ouverte pluridisciplinaire HAL, est destinée au dépôt et à la diffusion de documents scientifiques de niveau recherche, publiés ou non, émanant des établissements d'enseignement et de recherche français ou étrangers, des laboratoires publics ou privés. 


\title{
ASYMPTOTIC PERFORMANCE OF THE LOW RANK ADAPTIVE NORMALIZED MATCHED FILTER IN A LARGE DIMENSIONAL REGIME
}

\author{
Alice Combernoux ${ }^{1}$, Frédéric Pascal ${ }^{1}$, Guillaume Ginolhac ${ }^{2}$, Marc Lesturgie ${ }^{1}$ \\ ${ }^{1}$ SONDRA - Supelec, Plateau du Moulon, 3 rue Joliot-Curie, F-91192 Gif-sur-Yvette Cedex, France \\ ${ }^{2}$ LISTIC - Polytech Annecy-Chambéry, 5 chemin de Bellevue, 74944 Annecy-le-Vieux Cedex, France
}

\begin{abstract}
The paper addresses the problem of approximating the detector distribution used in target detection embedded in a disturbance composed of a low rank Gaussian noise and a white Gaussian noise. In this context, it is interesting to use an adaptive version of the Low Rank Normalized Matched Filter (LR-ANMF) detector, which is a function of the estimated projector onto the low rank noise subspace. We will show that the traditional approximation of the LR-ANMF detector distribution is not always the better one. In this paper, we propose to perform its limits when the number of secondary data $K$ and the data dimension $m$ both tend to infinity at the same rate $m / K \rightarrow c \in(0, \infty)$. Then, we give the theoretical distributions of these limits in the large dimensional regime and approximate the LR-ANMF detector distribution by them. The comparison of empirical and theoretical distributions on a jamming application shows the interest of our approach.
\end{abstract}

Index Terms - Low rank detection, Random matrix theory, Asymptotic distribution, Adaptive Normalized Matched Filter.

\section{INTRODUCTION}

In the context of target detection in a noise composed of a low rank (LR) Gaussian noise and an AWGN (Additive White Gaussian Noise), one could use the Low Rank Normalized Matched Filter (LR-NMF) detector [1] in order to exploit this low rank structure. Indeed, although its full rank version (NMF detector [2]) depends on the covariance matrix, the LR-NMF detector only requires the projector onto the LR noise subspace.

However, in practice, this projector and the covariance matrix are unknown and it is necessary to estimate them using $K$ secondary data which share the same properties as the tested data. Traditionally, the projector is estimated using the estimated covariance matrix: the Sample Covariance Matrix (SCM). Then, the adaptive detector is obtained substituting the true projector by the estimated one in the LR-NMF detector. The obtained detector is then named LR-ANMF (LR Adaptive NMF) detector. One also could note that it needs much less secondary data as its classical version (ANMF detector [3]) for equivalent performances in terms of filtering $[4,5]$, which is useful in practice.

Hence, engineers should want to calibrate their detector with an automatic tuning of the threshold in order to obtain a certain false alarm probability. In order to do this, they need the knowledge of the distribution of the employed detector, here the LR-ANMF detector. Unfortunately, its distribution is not known. That is why, we classically approximate it by the well-known distribution of the LR-NMF detector [1].

Nevertheless, depending on the data dimension $m$ and the number of secondary data $K$, this approximation could not be always the most appropriated. However, using the Random Matrix Theory (RMT), one could better approximate the distribution of the LRANMF detector. Indeed, it is composed of 3 quadratic forms and, under the large dimensional regime (i.e. $m, K \rightarrow \infty$ at the same rate $m / K \rightarrow c \in(0,+\infty))$, their limits differ and depend on $c$ and on the SCM model. Thus, we propose to study the LR-ANMF detector convergences when $m, K \rightarrow \infty$ at the same ratio under the two main data/SCM models considered in RMT. The first model is a basic model proposed by [6,7] where the multiplicity of the covariance matrix's eigenvalues grows with the dimension $m$ at a fixed ratio. The second data model, the spiked model introduced by [8] considers that the eigenvalues multiplicity corresponding to the low rank signal is fixed at the same value even if $m$ increases and leads to a deterministic limit of the quadratic forms [9]. Then, the distribution of the limits in the large dimensional regime will be established in an intermediate form which will be finalized in a forthcoming paper. They will allow to more precisely approximate the LR-ANMF detector distribution.

As an illustration of the interest of the proposed approximations, the jamming application is studied. The purpose of this application is to detect a target despite jammers which emit energy in order to disrupt the target signal. In this case, the LR Gaussian noise is composed of the different jammers. The simulations will compare the empirical distributions of the LR-NMF, the LR-ANMF and their RMT limits as well as their theoretical distributions.

The paper is organized as follows: Section 2 presents the problem statement and the definition of the LR-NMF and the LR-ANMF detectors. Section 3 contains the main theoretical contributions of this work, i.e. the limits of the LR-ANMF detector in the large dimensional regime for two models of covariance matrix and their theoretical distributions. Finally, Section 4 shows the jamming application which illustrates the obtained results.

Notations: An italic letter stands for a scalar quantity, boldface lowercase (uppercase) characters stand for vectors (matrices) and $(.)^{H}$ stands for the conjugate transpose. $\mathbf{I}_{N}$ is the $N \times N$ identity matrix. $\operatorname{diag}($.$) denotes the diagonalization operator such as$ $(\mathbf{A})_{i, i}=(\operatorname{diag}(\mathbf{a}))_{i, i}=(\mathbf{a})_{i}$ and equal to zero otherwise. $\llbracket a, b \rrbracket$ is the set defined by $\left\{x \in \mathbb{Z}: a \leqslant x \leqslant b, \forall(a, b) \in \mathbb{Z}^{2}\right\} . X \perp Y$ for the random variables $X$ and $Y$ stands for independence between them. The abbreviation iid stems for independent and identically distributed.

\section{LOW RANK DETECTION}

\subsection{Problem formulation}

In an observation vector $\boldsymbol{x} \in \mathbb{C}^{m \times 1}$, the problem consists in detecting a complex signal $\boldsymbol{d}$ corrupted by an additive noise $\boldsymbol{c}+\boldsymbol{n}$. This detection problem can be formulated as the following binary 
hypothesis test:

$$
\left\{\begin{array}{lll}
\mathcal{H}_{0}: \boldsymbol{x}=\boldsymbol{c}+\boldsymbol{n} & \boldsymbol{x}_{k}=\boldsymbol{c}_{k}+\boldsymbol{n}_{k}, & k \in \llbracket 1, K \rrbracket \\
\mathcal{H}_{1}: \boldsymbol{x}=\boldsymbol{d}+\boldsymbol{c}+\boldsymbol{n} & \boldsymbol{x}_{k}=\boldsymbol{c}_{k}+\boldsymbol{n}_{k}, & k \in \llbracket 1, K \rrbracket
\end{array}\right.
$$

where the target response $\boldsymbol{d}=\alpha \boldsymbol{a}\left(\boldsymbol{\Theta}_{\boldsymbol{d}}\right), \alpha$ is the unknown amplitude and $\boldsymbol{a}\left(\boldsymbol{\Theta}_{\boldsymbol{d}}\right)$ is the target localization steering vector. The $\boldsymbol{x}_{k}$ 's $\in \mathbb{C}^{m \times 1}$ correspond to the $K$ iid signal-free data (secondary data) used for the noise parameters estimation. This noise is composed of an AWGN, $\boldsymbol{n}_{k} \in \mathbb{C}^{m \times 1}$ (resp. $\left.\boldsymbol{n}\right) \sim \mathcal{C N}\left(\mathbf{0}, \sigma^{2} \mathbf{I}_{m}\right)$ and a LR Gaussian noise $\boldsymbol{c}_{k} \in \mathbb{C}^{m \times 1}$ (resp. $\boldsymbol{c}$ ), modeled by a zero-mean complex Gaussian vector with a normalized covariance matrix $\mathbf{C}$ $(\operatorname{tr}(\mathbf{C})=m)$, i.e. $\boldsymbol{c}_{k} \sim \mathcal{C N}(\mathbf{0}, \mathbf{C})$. Consequently, the covariance matrix of the secondary data is $\mathbf{R}=\mathbf{C}+\sigma^{2} \mathbf{I}_{m} \in \mathbb{C}^{m \times m}$. Moreover, considering a LR Gaussian noise, one has $\operatorname{rank}(\mathbf{C})=r \ll m$ and hence, the eigendecomposition of $\mathbf{C}$ is $\mathbf{C}=\sum_{i=1}^{r} \gamma_{i} \boldsymbol{u}_{i} \boldsymbol{u}_{i}^{H}$ where $\gamma_{i}$ and $\boldsymbol{u}_{i}$ are the non-zero eigenvalues and the associated eigenvectors of $\mathbf{C}$ respectively and unknown in practice. This leads to $\mathbf{R}=\sum_{i=1}^{m} \lambda_{i} \boldsymbol{u}_{i} \boldsymbol{u}_{i}^{H}$ with $\lambda_{1}=\gamma_{1}+\sigma^{2}>\cdots>\lambda_{r}=\gamma_{r}+\sigma^{2}>$ $\lambda_{r+1}=\cdots=\lambda_{m}=\sigma^{2}$. Then, we define the projector onto the LR Gaussian noise subspace $\boldsymbol{\Pi}_{\mathrm{c}}$ and the projector onto the subspace orthogonal to the LR Gaussian noise subspace $\Pi_{\mathrm{c}}^{\perp}$ :

$$
\left\{\begin{array}{l}
\boldsymbol{\Pi}_{\mathrm{c}}=\sum_{i=1}^{r} \boldsymbol{u}_{i} \boldsymbol{u}_{i}^{H} \\
\boldsymbol{\Pi}_{\mathrm{c}}^{\perp}=\mathbf{I}_{m}-\boldsymbol{\Pi}_{\mathrm{c}}=\sum_{i=r+1}^{m} \boldsymbol{u}_{i} \boldsymbol{u}_{i}^{H}
\end{array}\right.
$$

\subsection{LR-NMF and LR-ANMF detectors}

A filtering preprocessing is applied on the observation vector $\boldsymbol{x}$ in order to remove the LR Gaussian noise. This leads to the following standard signal detection problem:

$$
\left\{\begin{array}{l}
\mathcal{H}_{0}: \boldsymbol{r}=\mathbf{U}_{0}^{H} \boldsymbol{x}=\boldsymbol{n}_{0} \\
\mathcal{H}_{1}: \boldsymbol{r}=\mathbf{U}_{0}^{H} \boldsymbol{x}=\boldsymbol{d}_{0}+\boldsymbol{n}_{0}
\end{array}\right.
$$

where $\mathbf{U}_{0}=\left[\boldsymbol{u}_{r+1}, \cdots, \boldsymbol{u}_{m}\right]$. Solving this detection problem and considering the white noise power of $\boldsymbol{n}_{0}$ as unknown leads to the LR-NMF [1] detector:

$$
\Lambda(\boldsymbol{\Theta})=\frac{\left|\boldsymbol{a}(\boldsymbol{\Theta})^{H} \boldsymbol{\Pi}_{\mathrm{c}}^{\perp} \boldsymbol{x}\right|^{2}}{\left(\boldsymbol{a}(\boldsymbol{\Theta})^{H} \boldsymbol{\Pi}_{\mathrm{c}}^{\perp} \boldsymbol{a}(\boldsymbol{\Theta})\right)\left(\boldsymbol{x}^{H} \boldsymbol{\Pi}_{\mathrm{c}}^{\perp} \boldsymbol{x}\right)} \underset{\mathcal{H}_{0}}{\stackrel{\mathcal{H}_{1}}{\gtrless} \delta}
$$

where $\delta$ stands for the detection threshold.

However, in practice, the covariance matrix $\mathbf{R}$ and the projector $\Pi_{\mathrm{c}}^{\perp}$ are unknown and have to be estimated. Classically, the Sample Covariance Matrix (SCM) is computed from the $K$ secondary data and can be written as $\hat{\mathbf{R}}_{\mathrm{SCM}}=\frac{1}{K} \sum_{k=1}^{K} \boldsymbol{x}_{k} \boldsymbol{x}_{k}^{H}=\sum_{i=1}^{m} \hat{\lambda}_{i} \hat{\boldsymbol{u}}_{i} \hat{\boldsymbol{u}}_{i}^{H}$ where $\hat{\lambda}_{i}$ and $\hat{\boldsymbol{u}}_{i}$ are the SCM eigenvalues and eigenvectors respectively. Finally, the estimated projectors are:

$$
\begin{aligned}
\hat{\boldsymbol{\Pi}}_{\mathrm{c}, \mathrm{SCM}} & =\sum_{i=1}^{r} \hat{\boldsymbol{u}}_{i} \hat{\boldsymbol{u}}_{i}^{H} \\
\hat{\mathbf{\Pi}}_{\mathrm{c}, \mathrm{SCM}}^{\perp} & =\mathbf{I}_{m}-\hat{\boldsymbol{\Pi}}_{\mathrm{c}, \mathrm{SCM}}=\sum_{i=r+1}^{m} \hat{\boldsymbol{u}}_{i} \hat{\boldsymbol{u}}_{i}^{H},
\end{aligned}
$$

leading to the LR-ANMF detector, written as:

$$
\hat{\Lambda}(\boldsymbol{\Theta})=\frac{\left|\boldsymbol{a}(\boldsymbol{\Theta})^{H} \hat{\boldsymbol{\Pi}}_{\mathrm{c}, \mathrm{SCM}}^{\perp} \boldsymbol{x}\right|^{2}}{\left(\boldsymbol{a}(\boldsymbol{\Theta})^{H} \hat{\boldsymbol{\Pi}}_{\mathrm{c}, \mathrm{SCM}}^{\perp} \boldsymbol{a}(\boldsymbol{\Theta})\right)\left(\boldsymbol{x}^{H} \hat{\boldsymbol{\Pi}}_{\mathrm{c}, \mathrm{SCM}}^{\perp} \boldsymbol{x}\right)} \underset{\mathcal{H}_{0}}{\stackrel{\mathcal{H}_{1}}{\gtrless}} \xi
$$

where $\xi$ stands for the detection threshold.

\section{STATISTICAL ANALYSIS OF THE LR-ANMF DETECTOR}

This section contains the main theoretical contributions of this work. First, the limits of the LR-ANMF detector are established for different covariance matrix models in the large dimensional regime. Then, the corresponding asymptotic distributions are derived.

\subsection{Convergences}

It is well known that the LR-ANMF is consistent for a fixed $m$ when $K \rightarrow \infty$, i.e. it converges to the LR-NMF. However, it has been proved in [10], using RMT techniques, that the LR-ANMF converges to another undetailed quantity when both $m, K \rightarrow \infty$ with $m / K \rightarrow c$. This quantity depends on the covariance matrix model (and of course on $c$ ). This paper focuses on the two most prevalent models: the model proposed by [6, 7] (named GMUSIC model in the remainder of the paper) and the spiked model $[8,9,11]$.

The GMUSIC model considers that the multiplicity of the eigenvalues of $\mathbf{R}$ increases with $m$ at a fixed ratio. On the other hand, since our problem consists in a LR Gaussian noise and a white Gaussian noise, the spiked model better fits with our data as it considers the signal of interest as a sum of $r$ fixed rank perturbations, e.g. there are $r$ eigenvalues of fixed multiplicity as $m$ increases. To prove the convergences, we assume the following standard hypotheses.

(As1) $\mathbf{R}$ has uniformly bounded spectral norm $\forall m \in \mathbb{N}^{*}$, i.e. $\forall i \in \llbracket 1, m \rrbracket, \lambda_{i}<\infty$.

(As2) The vectors $\boldsymbol{s}_{1}, \boldsymbol{s}_{2} \in \mathbb{C}^{m \times 1}$ used in the quadratic forms (here $\boldsymbol{a}(\Theta)$ and $\boldsymbol{x}$ ) have uniformly bounded Euclidean norm $\forall m \in \mathbb{N}^{*}$. In practice, they will be fixed to 1 .

(As3) Let $\mathbf{Y} \in \mathbb{C}^{m \times K}$ having iid entries $y_{i j} \sim \mathcal{C N}(0,1)$, absolutely continuous and with $\mathbb{E}\left[\left|y_{i j}\right|^{8}\right]<\infty$.

(As4) Let $\mathbf{Y} \in \mathbb{C}^{m \times K}$ defined as in (As3). Then, its distribution is invariant by left multiplication by a deterministic unitary matrix. Moreover, the eigenvalues empirical distribution function of $\frac{1}{K} \mathbf{Y} \mathbf{Y}^{H}$ almost surely (a.s.) converges to the Marčenko-Pastur distribution [12] with support $\left[(1-\sqrt{c})^{2},(1+\sqrt{c})^{2}\right]$.

(As5) The maximum (resp. minimum) eigenvalue of $\frac{1}{K} \mathbf{Y} \mathbf{Y}^{H}$ tends a.s. to $(1+\sqrt{c})^{2}$ (resp. to $\left.(1-\sqrt{c})^{2}\right)$.

As a comment, $\mathbf{Y}$ can be related to $\hat{\mathbf{R}}_{\mathrm{SCM}}$ with the relation $\hat{\mathbf{R}}_{\mathrm{SCM}}=\mathbf{R}^{1 / 2}\left(\frac{1}{K} \mathbf{Y} \mathbf{Y}^{H}\right) \mathbf{R}^{1 / 2}$. Finally, the last assumption, namely the separation condition depends on the model of the SCM:

(As6-GMUSIC) $1 / c$ is lower bounded (see [6]).

(As6-SPIKED) The eigenvalues of $\mathbf{R}$ satisfy $\left|\lambda_{i}-1\right|>\sqrt{c}$, $\forall i \in \llbracket 1, r \rrbracket$.

Under (As1-As5, As6-GMUSIC), [6] proved that:

$$
\boldsymbol{s}_{1}^{H} \hat{\boldsymbol{\Pi}}_{\mathrm{c}, \mathrm{SCM}}^{\perp} \boldsymbol{s}_{2} \underset{\substack{m, K \rightarrow \infty \\ m / K \rightarrow c<\infty}}{\stackrel{\text { a.s. }}{\longrightarrow}} \boldsymbol{s}_{1}^{H} \overline{\boldsymbol{\Pi}}_{\mathrm{c}, \mathrm{G}}^{\perp} \boldsymbol{s}_{2}
$$

with $\overline{\boldsymbol{\Pi}}_{\mathrm{c}, \mathrm{G}}^{\perp}=\sum_{i=1}^{m} w_{i}^{\mathrm{G}} \boldsymbol{u}_{i} \boldsymbol{u}_{i}^{H}$ and

$$
w_{i}^{\mathrm{G}}= \begin{cases}1-\frac{1}{m-r-1} \sum_{n=1}^{r}\left(\frac{\sigma^{2}}{\lambda_{n}-\sigma^{2}}-\frac{\mu_{m}}{\lambda_{n}-\mu_{m}}\right) & \text { if } i>r \\ \frac{\sigma^{2}}{\lambda_{i}-\sigma^{2}}-\frac{\mu_{m}}{\lambda_{i}-\mu_{m}} & \text { else }\end{cases}
$$

where $\mu_{1} \geqslant \cdots \geqslant \mu_{m}$ are the eigenvalues of $\operatorname{diag}(\boldsymbol{\lambda})-\frac{c}{m} \sqrt{\boldsymbol{\lambda}} \sqrt{\boldsymbol{\lambda}}^{T}$, $\boldsymbol{\lambda}=\left[\lambda_{1}, \cdots, \lambda_{m}\right]^{T}$ and $\sigma^{2}$ is the white noise power. 
Under (As1-As5, As6-SPIKED), [9] proved that

$$
\boldsymbol{s}_{1}^{H} \hat{\boldsymbol{\Pi}}_{\mathrm{c}, \mathrm{SCM}}^{\perp} \boldsymbol{s}_{2} \underset{\substack{m, K \rightarrow \infty \\ m / K \rightarrow c<\infty}}{\stackrel{\text { a.s. }}{\rightarrow}} \boldsymbol{s}_{1}^{H} \overline{\boldsymbol{\Pi}}_{\mathrm{c}, \mathrm{S}}^{\perp} \boldsymbol{s}_{2}
$$

with $\overline{\boldsymbol{\Pi}}_{\mathrm{c}, \mathrm{S}}^{\perp}=\sum_{i=1}^{m} w_{i}^{\mathrm{S}} \boldsymbol{u}_{i} \boldsymbol{u}_{i}^{H}$ and

$$
w_{i}^{\mathrm{S}}= \begin{cases}1 & \text { if } i>r \\ 1-\frac{1-c\left(\lambda_{i}-1\right)^{-2}}{1+c\left(\lambda_{i}-1\right)^{-1}} & \text { else }\end{cases}
$$

Thus, using the Slutsky's theorem and Eqs.(7) and (9), one obtains the limits of the LR-ANMF when $m, K \rightarrow \infty$ with $m / K \rightarrow c$ :

\section{GMUSIC model}

$\hat{\Lambda}(\boldsymbol{\Theta}) \underset{\substack{m, K \rightarrow \infty \\ m / K \rightarrow c<\infty}}{\stackrel{\mathbb{P}}{\longrightarrow}} \bar{\Lambda}^{\mathrm{G}}(\boldsymbol{\Theta})=\frac{\left|\boldsymbol{a}(\boldsymbol{\Theta})^{H} \overline{\boldsymbol{\Pi}}_{\mathrm{c}, \mathrm{G}}^{\perp} \boldsymbol{x}\right|^{2}}{\left(\boldsymbol{a}(\boldsymbol{\Theta})^{H} \overline{\boldsymbol{\Pi}}_{\mathrm{c}, \mathrm{G}}^{\perp} \boldsymbol{a}(\boldsymbol{\Theta})\right)\left(\boldsymbol{x}^{H} \overline{\boldsymbol{\Pi}}_{\mathrm{c}, \mathrm{G}}^{\perp} \boldsymbol{x}\right)}$

Spiked model

$$
\hat{\Lambda}(\boldsymbol{\Theta}) \underset{\substack{m, K \rightarrow \infty \\ m / K \rightarrow c<\infty}}{\stackrel{\mathbb{P}}{\longrightarrow}} \bar{\Lambda}^{\mathrm{S}}(\boldsymbol{\Theta})=\frac{\left|\boldsymbol{a}(\boldsymbol{\Theta})^{H} \overline{\boldsymbol{\Pi}}_{\mathrm{c}, \mathrm{S}}^{\perp} \boldsymbol{x}\right|^{2}}{\left(\boldsymbol{a}(\boldsymbol{\Theta})^{H} \overline{\boldsymbol{\Pi}}_{\mathrm{c}, \mathrm{S}}^{\perp} \boldsymbol{a}(\boldsymbol{\Theta})\right)\left(\boldsymbol{x}^{H} \overline{\boldsymbol{\Pi}}_{\mathrm{c}, \mathrm{S}}^{\perp} \boldsymbol{x}\right)}
$$

Notice that, since the convergence occurs for a given model and when $m \rightarrow \infty$, these limits are expected to be better approximations of the LR-ANMF than the LR-NMF. This will be highlighted in the simulations section.

\subsection{Asymptotic distributions}

This section is devoted to the derivation of the theoretical distributions of the LR-ANMF limits, under $\mathcal{H}_{0}$, permitting to obtain the threshold for a fixed false alarm probability. These distributions will allow to have a better approximation of the LR-ANMF distribution than the classical one given by the LR-NMF distribution [1]. This is of utmost interest for instance, to obtain a better false alarm regulation. This is the purpose of the following theorem.

Theorem 1. Let $w$ (resp. $\bar{\Lambda}(\boldsymbol{\Theta}))$ stands for $w^{\mathrm{G}}$ or $w^{\mathrm{S}}\left(\right.$ resp. $\bar{\Lambda}^{\mathrm{G}}(\boldsymbol{\Theta})$ or $\bar{\Lambda}^{\mathrm{S}}(\boldsymbol{\Theta})$ ) according to the underlying model (GMUSIC or spiked). Then, under $\mathcal{H}_{0},($ As1-As5) and the separation condition (As6), $\bar{\Lambda}(\Theta)$ shares the distribution of the following quantity:

$$
\frac{\sum_{i=1}^{m} \alpha_{i} X_{i}+2 \sum_{\substack{i, j=1 \\ i<j}}^{m} \beta_{i j}\left(\Re\left(z_{i j}\right) Y_{i j}+\Im\left(z_{i j}\right) Z_{i j}\right)}{C \sum_{i=1}^{m} \gamma_{i} X_{i}}
$$

where

$$
\left\{\begin{array}{l}
X_{i} \stackrel{\text { iid }}{\sim} \chi_{i}^{2}(2) \\
Y_{i j} \sim \operatorname{Laplace}_{i j}^{Y}(0,1 / 2) \\
Z_{i j} \sim \text { Laplace }_{i j}^{Z}(0,1 / 2)
\end{array}\right.
$$

and, if we define $\mathbf{U}=\left[\boldsymbol{u}_{1}, \cdots, \boldsymbol{u}_{m}\right]$,

$$
\left\{\begin{array}{l}
\alpha_{i}=\frac{1}{2} w_{i}^{2} \lambda_{i}\left|\boldsymbol{a}(\boldsymbol{\Theta})^{H} \boldsymbol{u}_{i}\right|^{2} \\
\beta_{i j}=w_{i} w_{j} \sqrt{\lambda_{i} \lambda_{j}} \\
z_{i j}=\boldsymbol{u}_{i}^{H} \boldsymbol{a}(\boldsymbol{\Theta}) \boldsymbol{a}(\boldsymbol{\Theta})^{H} \boldsymbol{u}_{j} \\
\gamma_{i}=w_{i} \lambda_{i} \\
C=\frac{1}{2}\left\|\operatorname{diag}\left(\left[w_{1}, \cdots, w_{m}\right]\right) \mathbf{U}^{H} \boldsymbol{a}(\boldsymbol{\Theta})\right\|_{2}^{2}
\end{array}\right.
$$

Moreover, $Y_{i j}$ (resp. $Z_{i j}$ ) is independent of $Y_{i^{\prime} j^{\prime}}$ (resp. $\left.Z_{i^{\prime} j^{\prime}}\right)$ for $\{i, j\} \neq\left\{i^{\prime}, j^{\prime}\right\}$ and $Y_{i j}$ and $X_{i}, Z_{i j}$ and $X_{i}$, and $Y_{i j}$ and $Z_{i j}$ are uncorrelated (but not independent) for all $i, j$.
Proof. Due to the lack of space, the detailed proof is omitted and will appear in a forthcoming paper. However, the proof outline is as follows. First, some rotations are applied on the different quadratic forms appearing in $\bar{\Lambda}(\boldsymbol{\Theta})$ in order to obtain functions of standard complex Gaussian random variables. Then, after some algebraic manipulations, $\bar{\Lambda}(\Theta)$ can be reduced to the form given by Eq.(13).

Observe that the distribution of Eq.(13) provides a distribution completely independent of the observation vectors $\boldsymbol{x}$ and $\boldsymbol{x}_{k}$ 's. Although this relationship is exploited in the paper in order to better approximate the distribution of the LR-ANMF, this result can be interpreted as follows. An immediate consequence of Theorem 1 is to automatically and theoretically regulate the false alarm probability or equivalently the threshold $\xi$ (Eq.(6)) as a function of $\Theta$ and the ratio $c$ (abusively equal to $m / K$ ) which is not the case with the LR-NMF distribution.

\section{SIMULATIONS}

In this section, the limits of the LR-ANMF and the corresponding distributions under $\mathcal{H}_{0}$ are analysed through Monte-Carlo simulations.

\subsection{Parameters}

To illustrate the interest of these convergences, the jamming application is chosen. The main purpose of this application is to detect a target thanks to a uniform linear antenna composed of $m$ sensors despite the presence of jamming. The response of the jamming, $\boldsymbol{c}$ is composed of signals similar to the target response. In this section, except for the convergences when $m, K \rightarrow \infty$ at the same rate $c$, we choose $m=100$ in order to have a large number for the data dimension. Even if, in practice, this number could not be always realistic, it allows to highlight the interest of RMT where matrices are built from high dimensional observations. Moreover, such a value can be realistic in some applications as STAP [13].

Let us denote $\boldsymbol{\Theta}=\theta$ where $\theta$ is the AoA (Angle of Arrival). As the limits are studied under the $\mathcal{H}_{0}$ hypothesis, $\boldsymbol{d}=\mathbf{0}_{m \times 1}$. The jamming is composed of three synthetic targets with AoA $-20^{\circ}$, $0^{\circ}$ and $20^{\circ}$. Thus, the jamming (low rank Gaussian noise) has a rank equal to $r=3$. Then, the signal wavelength is $l_{0}=0.667 \mathrm{~m}$, the AWGN $\boldsymbol{n}$ power is $\sigma^{2}=1$ and the Jamming to Noise Ratio is $J N R_{\mathrm{dB}}=10 \mathrm{~dB}$. Finally, the theoretical covariance matrix of the total noise (jamming plus AWGN) is settled to

$$
\mathbf{R}=J N R \times \mathbf{U}_{c} \operatorname{diag}([6,2,1]) \mathbf{U}_{c}^{H}+\sigma^{2} \mathbf{I}_{m}
$$

with $\mathbf{U}_{\boldsymbol{c}}=\left[\boldsymbol{u}_{1} \cdots \boldsymbol{u}_{r}\right]$

\subsection{Empirical cumulative density functions}

We will first observe the empirical cumulative density function (ECDF) of $\hat{\Lambda}$ and its different limits: $\Lambda$ (Eq.(4)), $\bar{\Lambda}^{\mathrm{G}}$ (Eq.(11)) and $\bar{\Lambda}^{\mathrm{S}}$ (Eq.(12)) under the hypothesis $\mathcal{H}_{0}$. Then, the distributions of $\bar{\Lambda}^{\mathrm{G}}$ and $\bar{\Lambda}^{\mathrm{S}}$ (Eq.(13)) will be plotted and compared to the ECDFs to validate the results provided in Theorem 1 as well as to illustrate the interest of using the proposed limits instead of the classical LRNMF. In order to observe the pertinence of the convergences and the distributions, the quantities are plotted for two different values of $K$ ( $K=2 r$ and $K=2 m)$ and two different values of $\theta\left(\theta=20^{\circ}\right.$ i.e. exactly on the jamming angle and $\theta=25^{\circ}$ i.e. far from the jamming). On all the figures, the red color (resp. the blue color) corresponds to the case where $K=2 r$ (resp. $K=2 m$ ) for the different studied scenarios while the thick black curves correspond to the LR-NMF case (which does not depend on $K$ ). 
We are first interested in Fig. 1 where the ECDFs of $\hat{\Lambda}$ (triangles), $\Lambda, \bar{\Lambda}^{\mathrm{G}}$ (stars) and $\bar{\Lambda}^{\mathrm{S}}$ (circles) are plotted for $\theta=20^{\circ}$ at the top and $\theta=25^{\circ}$ at the bottom. Thus we observe that, despite the ECDF of the LR-NMF $(\Lambda)$, the other ECDFs vary as a function of $K$, especially for $\theta=20^{\circ}$. We also note that $\bar{\Lambda}^{\mathrm{S}}$ (circles) is always close to $\hat{\Lambda}$ (triangles) contrary to $\bar{\Lambda}^{\mathrm{G}}$ (stars) for $K$ small and $\theta=20^{\circ}$, and $\Lambda$ (thick black line) for $\theta=20^{\circ}$. As a consequence, the LR-NMF detector is not a good approximation of the LR-ANMF detector and the spiked model seems to be the right model to use, i.e. $\bar{\Lambda}^{\mathrm{S}}$ is robust to the number $K$ and the value $\theta$. Finally, one could remark that when we are far from the jamming, all the curves are almost superimposed. The interest of our study is consequently mainly located when we are on or close to the jamming. However, the limits still remain useful far from the jamming.
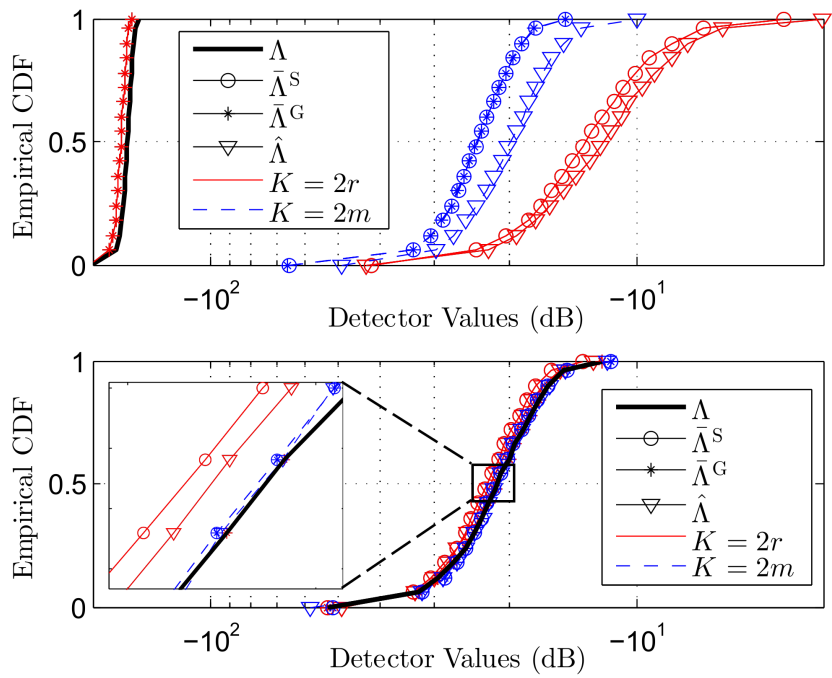

Fig. 1. Empirical cumulative density function of the limits of the LR-ANMF: (up) $\theta=20^{\circ}$, (down) $\theta=25^{\circ}$ with $J N R_{\mathrm{dB}}=10 \mathrm{~dB}$ over $10^{4}$ realisations.

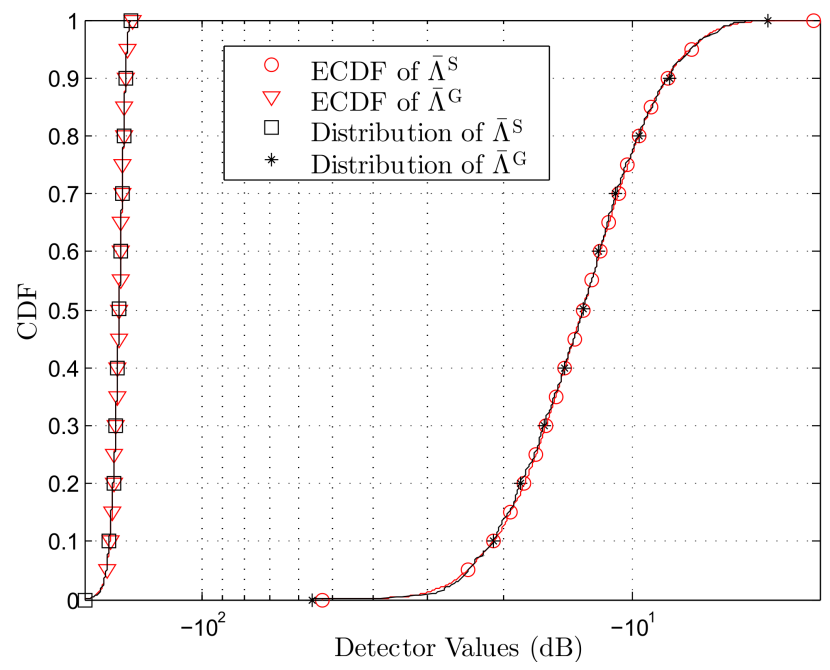

Fig. 2. Comparison between the ECDFs of the LR-ANMF RMT limits and their theoretical distribution for $\theta=20^{\circ}$ and $K=2 r$ over $10^{4}$ realisations.
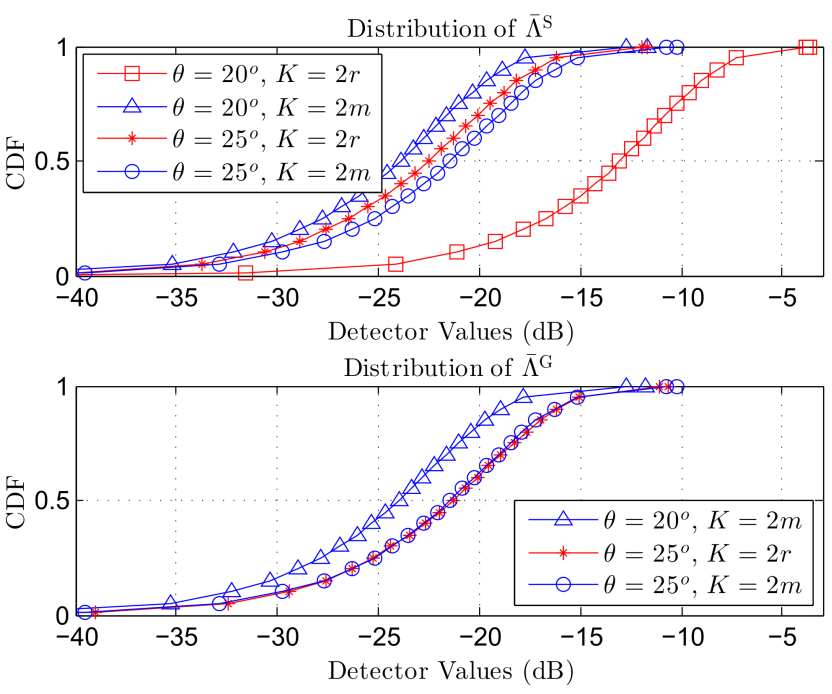

Fig. 3. CDF of the distributions of the limits of the LR-ANMF: $\bar{\Lambda}^{\mathrm{S}}$ (up) and $\bar{\Lambda}^{\mathrm{G}}$ (down) with $J N R_{\mathrm{dB}}=10 \mathrm{~dB}$ over $10^{4}$ realisations.

We then observe Fig. 2 where we compare the ECDFs of the LRANMF limits $\bar{\Lambda}^{\mathrm{G}}$ (triangles) and $\bar{\Lambda}^{\mathrm{S}}$ (circles) to their distributions (resp. stars and squares) from theorem 1 for $\theta=20^{\circ}$ and $K=2 r$. One could note that the ECDFs and their associated distributions are in perfect agreement which validate our theorem. Henceforth, the visualization of the ECDFs, dependent of the observation vector, is useless.

Thus, we finally observe Fig. 3 where the distributions of the RMT limits of $\hat{\Lambda}, \bar{\Lambda}^{\mathrm{G}}$ and $\bar{\Lambda}^{\mathrm{S}}$, are plotted at the top and at the bottom respectively. For visual readability reasons, the $\bar{\Lambda}^{\mathrm{G}}$ distribution for $\theta=20^{\circ}$ and $K=2 r$ is not plotted in Fig. 3 but can be observed in Fig.2. Then, the observations concerning the dependence of $\bar{\Lambda}^{\mathrm{G}}$ and $\bar{\Lambda}^{\mathrm{S}}$ on the ratio $c$ (abusively equal to $m / K$ ) are the same as those previously exposed with the ECDFs in Fig.1.

\section{CONCLUSION}

In this paper, we proposed to study the asymptotic performances of the LR-ANMF detector. That is why, using RMT tools, we performed its convergences and their theoretical distributions when both the number of secondary data $K$ and the data dimension $m$ tend to infinity at the same rate with 2 models of covariance matrix: the GMUSIC model and the spiked model. The obtained distributions allow to approximate the LR-ANMF distribution. Thus, we observed that the spiked model better describes the performances of the LRANMF detector as a function of $K$ than the LR-NMF detector and the GMUSIC model and is robust to the variations of the AoA and $K$. Finally, this paper allowed to conclude that the spiked model has to be used in a such application and that RMT tools are highly useful to obtain detector performance as a function of $K$ or $c$.

In the next future, we would like to determine the theoretical distribution of the limit of the LR-ANMF detector without contribution of several random variables when both $m, K \rightarrow \infty$ at the same rate $c$ for the different models of covariance matrix exposed in this paper. It would allows us to theoretically determine the distribution of the false alarm probability as a function of the threshold. We will also be interested in the derivation of the detection probability. 


\section{REFERENCES}

[1] M. Rangaswamy, F.C. Lin, and K.R. Gerlach, "Robust adaptive signal processing methods for heterogeneous radar clutter scenarios," Signal Processing, vol. 84, pp. 1653 - 1665, 2004.

[2] L.L. Scharf and B. Friedlander, "Matched subspace detectors," IEEE Trans. on Sig. Proc., vol. 42, pp. 2146 - 2157, 1994.

[3] L.L. Scharf and L.T. Mac Worther, "Adaptive matched subspace detector and adaptive coherence," Proc. of 30th Asilomar Conf. Signals, Syst., Comput., 1996.

[4] I. Kirstein and D. Tufts, "Adaptive detection using a low rank approximation to a data matrix," IEEE Trans. on Aero. and Elec. Syst., vol. 30, pp. 55 - 67, 1994.

[5] A. Haimovich, "Asymptotic distribution of the conditional signal-to-noise ratio in an eigenanalysis-based adaptive array," IEEE Trans. on Aero. and Elec. Syst., vol. 33, pp. 988 - 997, 1997.

[6] X. Mestre, "Improved estimation of eigenvalues and eigenvectors of covariance matrices using their sample estimates," IEEE Transactions on Information Theory, vol. 54, no. 11, pp. 5113 - 5129, November 2008.

[7] X. Mestre and M.A. Lagunas, "Modified subspace algorithms for DoA estimation with large arrays," IEEE Transactions on Information Theory, vol. 56, no. 2, pp. 598 - 614, February 2008.

[8] I. Johnstone, "On the distribution of the largest principal component," The Annals of Statistics, vol. 29, no. 2, pp. 295 - 327, 2001.

[9] R. Couillet and W. Hachem, "Fluctuations of spiked random matrix models and failure diagnosis in sensor networks," IEEE Transactions on Information Theory, vol. 59, no. 1, pp. 509 525, 2013.

[10] A. Combernoux, F. Pascal, G. Ginolhac, and M. Lesturgie, "Random matrix theory applied to low rank STAP detection," EUSIPCO, Sept. 2013, http://hal.archives-ouvertes.fr/hal00871217.

[11] J. Baik and Jack W. Silverstein, "Eigenvalues of large sample covariance matrices of spiked population models," Journal of Multivariate Analysis, vol. 97, pp. 1643 - 1697, 2006.

[12] V.A. Marčenko and L.A. Pastur, "Distributions of eigenvalues for some set of random matrices," Math USSR-Sbornik, vol. 1, no. 4, pp. 457 - 483, April 1967.

[13] J. Ward, "Space-time adaptive processing for airborne radar," Tech. Rep., Lincoln Lab., MIT, Lexington, Mass., USA, December 1994. 